\title{
Self- and Collective Dynamics of Syndiotactic Poly(methyl methacrylate). A Combined Study by Quasielastic Neutron Scattering and Atomistic Molecular Dynamics Simulations
}

 \\ A. Wischnewski, $\stackrel{\perp}{\text { and D. }}$ Richter $^{\perp}$ \\ Donostia International Physics Center, Paseo Manuel de Lardizabal 4, 20018 San Sebastián, Spain; \\ Unidad Física de Materiales (CSIC-UPV/EHU), Apartado 1072, 20080 San Sebastián, Spain; \\ Departamento de Física de Materiales (UPV/EHU), Apartado 1072, 20080 San Sebastián, Spain; \\ Institut Laue-Langevin, BP 156, 38042 Grenoble Cedex 9, France; and Institut für \\ Festkörperforschung, Forschungszentrum Jülich GmbH, D-52425 Jülich, Germany
}

Received April 5, 2006; Revised Manuscript Received July 12, 2006

\begin{abstract}
We have investigated the molecular dynamics of syndiotactic poly(methyl methacrylate) well above the glass transition by combining quasielastic neutron scattering and fully atomistic computer simulations. The incoherent scattering measured by backscattering on a sample with deuterated ester methyl groups has revealed the single-particle motions of hydrogens in the main chain and in the $\alpha$-methyl groups. Moreover, with neutron spin-echo experiments on the fully deuterated sample we have accessed the collective motions at the two first maxima of the structure factor. The simulated cell, which has been previously validated regarding the structural properties [Genix, A.-C.; et al. Macromolecules 2006, 39, 3947], shows a dynamical behavior that, allowing a shift in temperature, reproduces very accurately all the experimental results. The combined analysis of both sets of data has shown that: (i) The segmental relaxation involving backbone atoms deviates from Gaussian behavior. (ii) The dynamics is extremely heterogeneous: in addition to the subdiffusion associated with the $\alpha$-process and the methyl group rotations, we have found indications of a rotational motion of the ester side group around the main chain. (iii) At a given momentum transfer and depending on the molecular groups considered, the time scales for collective motion are spread over about 1 order of magnitude, the correlations involving the main chain decaying much more slowly than those relating side groups. (iv) At the length scale characteristic for the overall periodicity of the system (that corresponding to the first structure factor peak), the experimentally observed collective dynamics relates to the backbone motions and is of interchain character; there, coherency effects are observed for all correlations, though side groups display weaker collectivity. (v) At the second structure factor peak, coherency remains only for correlations involving the main chains.
\end{abstract}

\section{Introduction}

Poly(methyl methacrylate) (PMMA) is a very well-known glass-forming polymer that displays a complex dynamical behavior. Among the different dynamical processes taking place in PMMA, we first mention those related to rotational motions of both methyl groups: (i) The low values of the energy barriers in the ester methyl group have allowed resolution of rotational tunneling by quasielastic neutron scattering; ${ }^{1,2}$ accordingly, its classical rotation is very fast. $^{2-6}$ (ii) Because of stronger hindrance, the rotation of the $\alpha$-methyl group occurs only at higher temperatures. ${ }^{7,8}$ Furthermore, other subglass and glass relaxation processes in PMMA have been studied for a long time mainly by dynamic mechanical analysis, dielectric spectroscopy, and advanced NMR spectroscopy. ${ }^{9-17}$ Especially in the temperature range close to and above the glass-transition temperature $\left(T_{\mathrm{g}} \approx 400 \mathrm{~K}\right)$, PMMA exhibits a complicated dynamics. A complex - and likely cooperative-secondary $\beta$ relaxation has been reported, which masks the pure $\alpha$-process when techniques such as mechanical or dielectric spectroscopy (e.g., ref 13) are used. The molecular motions responsible for the $\beta$-process have been extensively studied by using multidimensional solid-state NMR experiments. ${ }^{11}$ The corresponding

\footnotetext{
Donostia International Physics Center.

$\div$ CSIC-UPV/EHU.

$\S \mathrm{UPV} / \mathrm{EHU}$.

"Institut Laue-Langevin.

${ }^{\perp}$ Forschungszentrum Jülich $\mathrm{GmbH}$.
}

results led to the conclusion that this process relates to $\pi$-flips of the carboxyl side group which are coupled with rotational readjustments of the local chain axis with amplitude of about $\pm 20^{\circ}$ (rocking motion). Moreover, by using two-dimensional NMR spectroscopy on PMMA, Kuebler et al. ${ }^{12}$ reported that the $\beta$-relaxation largely influences the $\alpha$-relaxation time scale leading to a high mobility of the main chain. In agreement with these findings, Soldera et al. ${ }^{18}$ found a correlation between the dihedral angle motions of the backbone and the side groups by using molecular simulations. Thus, side groups and backbone motions seem to be intimately related in PMMA.

Quasielastic neutron scattering (QENS) offers deep insight into the dynamical processes of glass-forming systems. This technique allows the study of the dynamics at a molecular level with space/time resolution. In addition, because of the different interaction of neutrons with hydrogens and deuterons, it is possible to follow either single-particle motions - those of $\mathrm{H}$ in protonated samples - or collective dynamics - the pair-correlation functions involving deuterons, carbons, deuterons and carbons, etc., in fully deuterated samples. Up to the present date, the potential of QENS has not been fully exploited to disentangle PMMA dynamical processes other than methyl group rotations. The three published QENS studies on PMMA dynamics close to and above the glass transition have been focused on the effect produced by blending with poly(ethylene oxide). In the first work, ${ }^{19}$ samples containing fully protonated PMMA were investigated. The homopolymer investigated as reference showed 
extremely strong deviations from the Gaussian approximation for the average self-correlation function of all PMMA hydrogens that were experimentally accessed. The second work ${ }^{8}$ did not report on the homopolymer dynamics. Finally, in the neutron spin-echo study on collective motion, ${ }^{20}$ the temperature range was restricted to only two temperatures very close to $T_{\mathrm{g}}$ for pure PMMA.

Complementary to neutron scattering, fully atomistic molecular dynamics (MD) simulations have proven to be a very powerful technique to unravel the structural and dynamical features of glass-forming systems. In a previous paper, ${ }^{21}$ we have already used such a combination to decipher the local structure of syndiotactic PMMA. In this work, we address the dynamical behavior of this polymer at temperatures well above the glass transition to contribute to the understanding of the molecular motions in this range. Experimentally, we have accessed two types of correlations: on one hand, backscattering measurements on a sample with deuterated ester methyl groups followed the single-particle motions of hydrogens in the mainchain and in the $\alpha$-methyl groups. We have avoided the contributions from the ester hydrogens because, as we will see later, their dynamics is clearly faster than those of the rest of the hydrogens. On the other hand, with neutron spin-echo experiments on the fully deuterated sample we have accessed the collective motions at the two first maxima of the structure factor. At these positions we have also followed the temperature dependence of the dynamic structure factor down to $T_{\mathrm{g}}$. All these experimental results provide a very critical check for the MD simulation, which was previously validated regarding the structural properties. ${ }^{21}$ After having validated the dynamical features of the simulated cell, we could calculate functions that cannot easily be accessed experimentally (e.g., pair correlations involving particular molecular groups) and learn further about the detailed dynamics of this system.

The structure of this paper reflects the following strategy. First, after the introduction of the experimental and simulation details, the results of the QENS measurements are presented and analyzed. These are the basis for validating the simulated cell in the next section. With the combined information of experiments and simulations at our disposal, we first discuss the self-motions and then collective dynamics. Finally, a short discussion addresses the comparison with other results from the literature, before summarizing the main conclusions of this work.

\section{Experimental Section}

A. Samples. Two PMMA samples (Polymer Source, Inc.) with different deuteration levels were investigated: fully deuterated $\left(\right.$ PMMA- $\left.d_{8}\right)$ and a protonated material including deuterated ester methyl groups (PMMA- $d_{3}$ ). The molecular weights and polydispersities were $M_{\mathrm{w}}=26800$ and $25300 \mathrm{~g} / \mathrm{mol}$ and $M_{\mathrm{w}} / M_{\mathrm{n}}=1.04$ and 1.09, respectively. The glass-transition temperatures determined by DSC were $T_{\mathrm{g}}=400 \mathrm{~K}$ for both polymers that were close to $80 \%$ syndiotactic.

B. Neutron Scattering. Information on the dynamics of a sample can be obtained by analyzing the change in energy $(\hbar \omega)$ experienced by neutrons scattered into a given solid angle between $\Omega$ and $\Omega+$ $\mathrm{d} \Omega .^{22-25}$ The modulus of the momentum transfer $Q\left(\vec{Q}=\vec{k}_{\mathrm{f}}-\vec{k}_{\mathrm{i}}\right)$ is determined by the scattering angle $\theta$ and the wavelength of the incoming neutrons $\lambda$ as $Q=4 \pi \sin (\theta / 2) / \lambda$. The measured intensity as a function of $Q$ and energy transfer $\hbar \omega$ contains the incoherent and the coherent contributions, $I_{\mathrm{inc}}(Q, \omega)$ and $I_{\mathrm{coh}}(Q, \omega)$.

The incoherent intensity $I_{\text {inc }}(Q, \omega)$ is given by the product of the incoherent scattering cross section and the incoherent scattering function, $I_{\text {inc }}(Q, \omega)=\left(k_{\mathrm{f}} / k_{\mathrm{i}}\right) \sigma_{\text {inc }} S_{\text {inc }}(Q, \omega) /(4 \pi)$. The scattering function $S_{\text {inc }}(Q, \omega)$ is related via Fourier transformation with the intermediate incoherent scattering function, $S_{\mathrm{s}}(Q, t)$. Its double Fourier transform is the self-part of the Van Hove correlation function, $G_{\mathrm{s}}(r, t)$. In the classical limit, $G_{\mathrm{s}}(r, t)$ is the probability of a given nucleus to be at a distance $r$ from the position where it was located at a time $t$ before. Thus, incoherent scattering looks at correlations between the positions of the same nucleus at different times.

The coherent scattering revealing the dynamic structure factor $S_{\text {coh }}(Q, t)$ deals with relative positions of atomic pairs, i.e., the collective dynamics. Its total intensity is determined by the differential scattering cross section, $(\partial \sigma / \partial \Omega)_{\text {coh }}$, revealing the structural features. In an "ideal" diffraction experiment, $(\partial \sigma / \partial \Omega)_{\mathrm{coh}}$ would be identical to the static structure factor $S(Q)$, i.e., the $t=$ 0 value of the dynamic structure factor $S_{\text {coh }}(Q, t)$.

We note that, as NS spectrometers offer a limited energy resolution, the measured functions are affected by the instrumental resolution function, $R(Q, \omega) . R(Q, \omega)$ is the obtained spectrum when purely elastic ( $\hbar \omega=0)$ scattering events take place in the sample (i.e., it is the "image" of $\delta(\omega)$ ). It can usually be determined from the scattering of the sample at very low temperature, where all the dynamical processes are frozen. Thus, the experimentally accessed quantity has to be compared with the convolution of the model function and the resolution, $I_{\exp } \sim I(Q, \omega) \otimes R(Q, \omega)$.

1. Neutron Spin Echo. Neutron spin echo (NSE) is the neutron scattering technique offering the highest energy resolution. For each neutron individually the energy transfer in the scattering process is coded into its spin rotation. ${ }^{26}$ Applying precession magnetic fields before and after the scattering event, the polarization of the neutron depends only on the velocity difference of each neutron individually, irrespective of its initial velocity.

As a singular feature of NSE, it accesses the scattering functions in the time domain, i.e., the intermediate scattering functions. NSE measures a normalized scattering function $a^{26}$

$$
\tilde{S}_{\mathrm{NSE}}(Q, t)=\frac{I_{\mathrm{coh}}(Q, t)-\frac{1}{3} I_{\mathrm{inc}}(Q, t)}{I_{\mathrm{coh}}(Q, 0)-\frac{1}{3} I_{\mathrm{inc}}(Q, 0)}
$$

$I_{\text {coh }}(Q, t)$ and $I_{\text {inc }}(Q, t)$ are related via Fourier transformation to the coherent and incoherent scattering intensities, respectively. The time variable depends on the precession field $B$, the wavelength $\lambda$, and the length of the precession field $L: t \sim B L \lambda^{3}$.

In this work, we used the NSE spectrometer IN11 at the Institut Laue-Langevin (ILL, Grenoble) to investigate the fully deuterated sample. With $\lambda=5.5 \AA$ the accessed time range was $0.04 \mathrm{~ns} \leq t$ $\leq 8$ ns. The $Q$ values investigated were 0.8 and $1.9 \AA^{-1}$, where PMMA- $d_{8}$ shows the two first maxima in the static structure factor. $^{21}$ We performed measurements at 400, 425, 449, 475, 496 (only for $1.9 \AA^{-1}$ ), and $516 \mathrm{~K}$. Measuring times were of about $8 \mathrm{~h}$ per $Q$ and temperature. Direct deconvolution of the measured spectra from the experimental resolution was realized dividing by a measurement on the sample at $5 \mathrm{~K}$, which is representative of the instrumental response to a purely elastic process.

2. Backscattering. The PMMA- $d_{3}$ sample was investigated at the backscattering (BS) instrument at the Jülich FRJ-2 reactor. In a BS experiment, perfect crystals are used as monochromator and analyzers, and the instrumental resolution is optimized by using backscattering geometry at both crystals. These spectrometers work under inverse geometry condition; i.e., the energy of the detected neutrons is fixed to a given value while the energy of the incident neutrons is varied around that value. In our experiment the energy window was set to $-17 \mu \mathrm{eV} \leq \hbar \omega \leq 17 \mu \mathrm{eV}$. Using $\lambda=6.271 \AA$, we accessed 10 different $Q$ values in the range between 0.16 and $1.87 \AA^{-1}$. The energy resolution was $\delta E($ hwhm $) \approx 0.45 \mu \mathrm{eV}$ for the detectors corresponding to $Q>0.6 \AA^{-1}$ and slightly worse for the smaller scattering angles $\left(0.7 \mu \mathrm{eV}\right.$ for $\left.0.16 \AA^{-1}\right)$. The PMMA$d_{3}$ sample was positioned at $45^{\circ}$ with respect to the incident beam. The temperature investigated was $518 \mathrm{~K}$ with a measuring time of $14 \mathrm{~h}$. The experimental resolution function was obtained from measurements on the sample at $5 \mathrm{~K}$. The efficiency of the detectors was determined from comparison of the integrated intensities at 5 $\mathrm{K}$ with the neutron diffraction results on the same sample obtained 
with polarization analysis. ${ }^{21}$ Background corrections were performed by subtracting the scattering of the empty cell.

\section{Simulation Method}

The fully atomistic MD simulations were carried out by using the Insight and the Discover-3 module from Accelrys with the polymer consortium force field. ${ }^{27-29}$ A cubic cell containing four polymer chains of 25 monomers each was constructed at $580 \mathrm{~K}$ by means of the Amorphous Cell protocol ${ }^{30,31}$ with periodic boundary conditions. The tacticity of the chains was close to $80 \%$. The simulated cell was first equilibrated with density fixed to unity during $20 \mathrm{~ns}$, and after that, with a series of dynamics steps at fixed atmospheric pressure, the equilibrium density was reached. The cell finally had $25.16 \AA$ of side. The following simulations were carried out at $580 \mathrm{~K}$ in the NVT ensemble, where the cell size and the temperature of the sample are constant. During a first 1 ns run the new cell was still allowed to equilibrate. Afterward, a 1 ns run was launched collecting data every 0.01 ps. Two more successive runs of 2 and $20 \mathrm{~ns}$ were carried out, collecting data every 0.05 and 0.5 ps, respectively. A detailed explanation of the simulation method has been given in our previous paper on the structure of PMMA. ${ }^{21}$

From the atomic trajectories obtained in the simulations we have calculated several magnitudes based on the Van Hove correlation function. First, the self-part of this function, $G_{\mathrm{s}}(\vec{r}, t)$, corresponding to the single particle motions, has been computed. Assuming isotropic behavior, for a given atom of type $\alpha$ ( $\alpha$ can be, e.g., main-chain carbons, ester methyl hydrogens, etc.), $G_{\mathrm{s}}^{\alpha}(r, t)$ is obtained as

$$
G_{\mathrm{s}}^{\alpha}(r, t)=\left\langle\frac{1}{N_{\alpha}} \sum_{i \alpha=1}^{N_{\alpha}} \delta\left[r-\left|\vec{r}_{i \alpha}(t)-\vec{r}_{i \alpha}(0)\right|\right]\right\rangle
$$

where $r$ is the radial distance from a given particle, $N_{\alpha}$ is the number of particles of type $\alpha$, and $\vec{r}_{i \alpha}$ is the position vector of the $i$ th particle of kind $\alpha$. The average considers a large number of atomic configurations. As previously mentioned, $G_{\mathrm{s}}(r, t)$ is directly related to the incoherent scattering accessed in a neutron scattering experiment (that of the hydrogens dominates the scattering signal). From $G_{\mathrm{s}}^{\alpha}(r, t)$ the intermediate scattering function can be computed by Fourier transformation:

$$
S_{\mathrm{s}}^{\alpha}(Q, t)=\int_{0}^{\infty} 4 \pi r^{2} G_{\mathrm{s}}^{\alpha}(r, t) \frac{\sin (Q r)}{Q r} \mathrm{~d} r
$$

The pair correlations related to coherent scattering will also be addressed. The pair correlation function of the position of nuclei of types $\alpha$ and $\beta, g^{\alpha \beta}(r, t)$, is

$$
g^{\alpha \beta}(r, t)=\frac{1}{N}\left\langle\sum_{i \alpha, j \beta}^{N_{\alpha} N_{\beta}} \delta\left(r-\left|\vec{r}_{i \alpha}(t)-\vec{r}_{j \beta}(0)\right|\right)\right\rangle
$$

For $t=0$, this function reduces to the well-known radial distribution function. To calculate $g^{\alpha \beta}(r, t)$, all atoms within a sphere of radius $r$ centered in the position of a given atom are considered. It should be noted that when evaluating $\vec{r}_{i \alpha}(t)-$ $\vec{r}_{j \beta}(0) \mid$ it is essential to use the distance between the atom $i \alpha$ and its nearest image atom $j \beta$, which may lie in the simulated cell or in one of the surrounding image cells. Periodic boundary conditions limit the range of $r$ to no more than half the size of the side of the simulation cell. The procedure followed by us has been widely described in basic references (see, e.g., refs 32 and 33).
The results obtained will be discussed in the reciprocal space through the structure factors. In an analogous way as for the self-term (eq 3), the Fourier transform of $g^{\alpha \beta}(r, t)$ yields the pair correlation functions $a_{\alpha \beta}(Q, t)$ in $Q$-space. The experimentally accessible structure factor is obtained by adding up all the contributing correlations properly_weighted with the scattering lengths of the involved isotopes $\bar{b}_{\alpha}, \bar{b}_{\beta}$ :

$$
S_{\mathrm{coh}}(Q, t)=\sum_{\alpha, \beta} \bar{b}_{\alpha} \bar{b}_{\beta} a_{\alpha \beta}(Q, t)
$$

$\left(\bar{b}_{\mathrm{H}}=-3.7406 \mathrm{fm} ; \bar{b}_{\mathrm{D}}=6.671 \mathrm{fm} ; \bar{b}_{\mathrm{C}}=6.6511 \mathrm{fm} ; \bar{b}_{\mathrm{O}}=\right.$ $5.803 \mathrm{fm}$ ). The constraints due to periodic boundary conditions mentioned above imply that the minimum reliable value of $Q$ for the calculated structure factor is $Q \approx 4 \pi / L$, where $L$ is the side of the simulated cell. Since $C$ and D show nearly identical scattering lengths and that of $\mathrm{O}$-the minority component of PMMA-is not very different, for the PMMA- $d_{8}$ sample all nuclei are almost equally weighted in eq 5 . Therefore, the coherent scattering measured for this sample is a good approximation for the total structure factor. We note that in hydrogenated or partially deuterated samples the different weightings of the different atomic pair contributions provide partial structure factors.

\section{Experimental Results}

A. Neutron Spin Echo. For the PMMA- $d_{8}$ sample investigated by NSE the coherent signal clearly dominates the scattering. ${ }^{21}$ In addition, the weak incoherent contribution is reduced by $1 / 3$ by the NSE instrument, and the measured signal reveals directly the normalized dynamic structure factor of PMMA: $\quad \tilde{S}_{\mathrm{NSE}}(Q, t)=S_{\mathrm{coh}}(Q, t) / S_{\mathrm{coh}}^{\mathrm{NSE}}(Q, 0)$. As we will see below, the static value of $S_{\mathrm{coh}}(Q, t)$ measured by the NSE spectrometer, $S_{\mathrm{coh}}^{\mathrm{NSE}}(Q, 0)$, slightly differs from the right value $S_{\mathrm{coh}}(Q, 0)=S(Q)$.

The NSE experiments studied $\tilde{S}_{\mathrm{NSE}}(Q, t)$ at the two first maxima of $S(Q)$, namely 0.8 and $1.9 \AA^{-1} \cdot{ }^{21}$ The observed structure factors at both peak positions are shown in Figure 1 for the different temperatures investigated. From Figure 1a,b we note that the amplitude of the decay depends on the $Q$ value and temperature considered. This effect relates to the fast processes present in glass-forming systems that lead to a decay of the correlations at time scales below a picosecond. In the NSE window, they can be parametrized in terms of the DebyeWaller factor (DWF). Furthermore, as usually observed in glassforming polymers, ${ }^{34}$ the decays do not show exponential functional forms but are more stretched. They can be well described by a Kohlrausch-Williams - Watts (KWW) or stretched exponential function:

$$
\tilde{S}_{\mathrm{NSE}}(Q, t)=\mathrm{DWF} \exp \left[-\left(\frac{t}{\tau_{\mathrm{w}}}\right)^{\beta}\right]
$$

where $\beta$ is the stretching parameter accounting for the shape of the relaxation function $\left(0<\beta \leq 1\right.$ and, for polymers, $\left.\beta \approx 0.5^{34}\right)$, and $\tau_{\mathrm{w}}$ is the relaxation time that depends on $Q$ and temperature. Since the fitting parameters DWF and $\beta$ are strongly correlated, to determine the temperature dependence of the time scales and the shape of the relaxation functions, we have constructed master curves for each $Q$ value. Individual fits of the NSE data for each $Q$ and temperature allowed a first estimation of the amplitudes. After normalization of the curves by the such obtained DWF, they were superimposed for a reference temperature $T_{\mathrm{R}}\left(T_{\mathrm{R}}=475 \mathrm{~K}\right)$ by applying temperature-dependent shift factors to the time scale. The resulting master curves were 


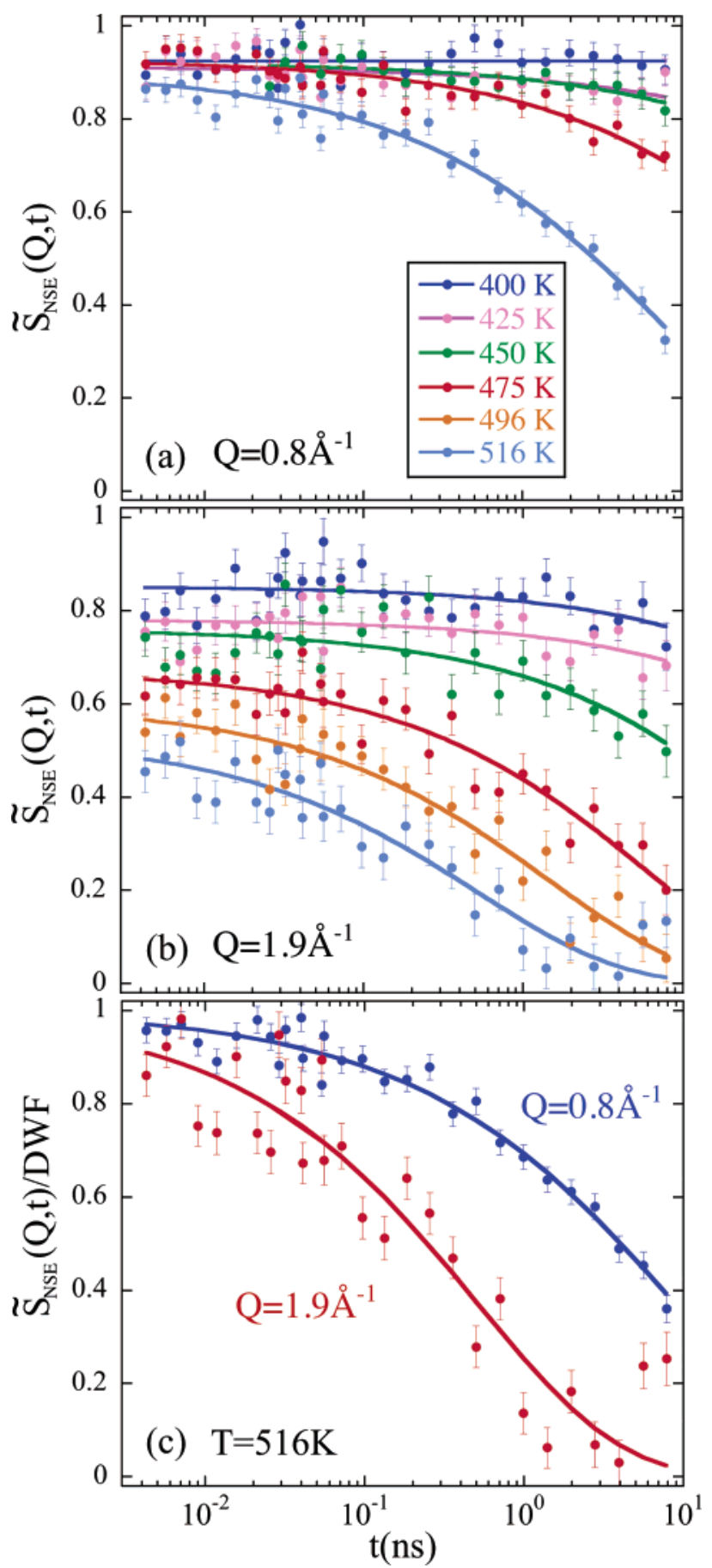

Figure 1. Dynamic structure factor of PMMA measured by IN11 at the two first maxima of $S(Q): Q=0.8 \AA^{-1}$ (a) and $Q=1.9 \AA^{-1}$ (b), and the different temperatures indicated in the inset of (a). In (c), the DWF-corrected curves are compared for both $Q$ values at $516 \mathrm{~K}$. The lines are KWW descriptions with $\beta\left(Q=0.8 \AA^{-1}\right)=0.46$ and $\beta(Q=$ $\left.1.9 \AA^{-1}\right)=0.49$

fitted to a KWW function, and the obtained $\beta$ parameter was used as starting value to repeat the procedure. This iterative approach converged very quickly, leading to the master curves shown in Figure 2. As we can appreciate, within the experimental uncertainties the results corresponding to different temperatures collapse quite nicely, indicating that the timetemperature superposition principle is rather well fulfilled by the PMMA dynamic structure factor. The values such obtained for $\beta$ are very similar for both $Q$ 's ( $\beta=0.46$ for $Q=0.8 \AA^{-1}$ and $\beta=0.49$ for $\left.Q=1.9 \AA^{-1}\right)$. As can be seen from Figure 1 , by fixing these stretching parameters very good individual
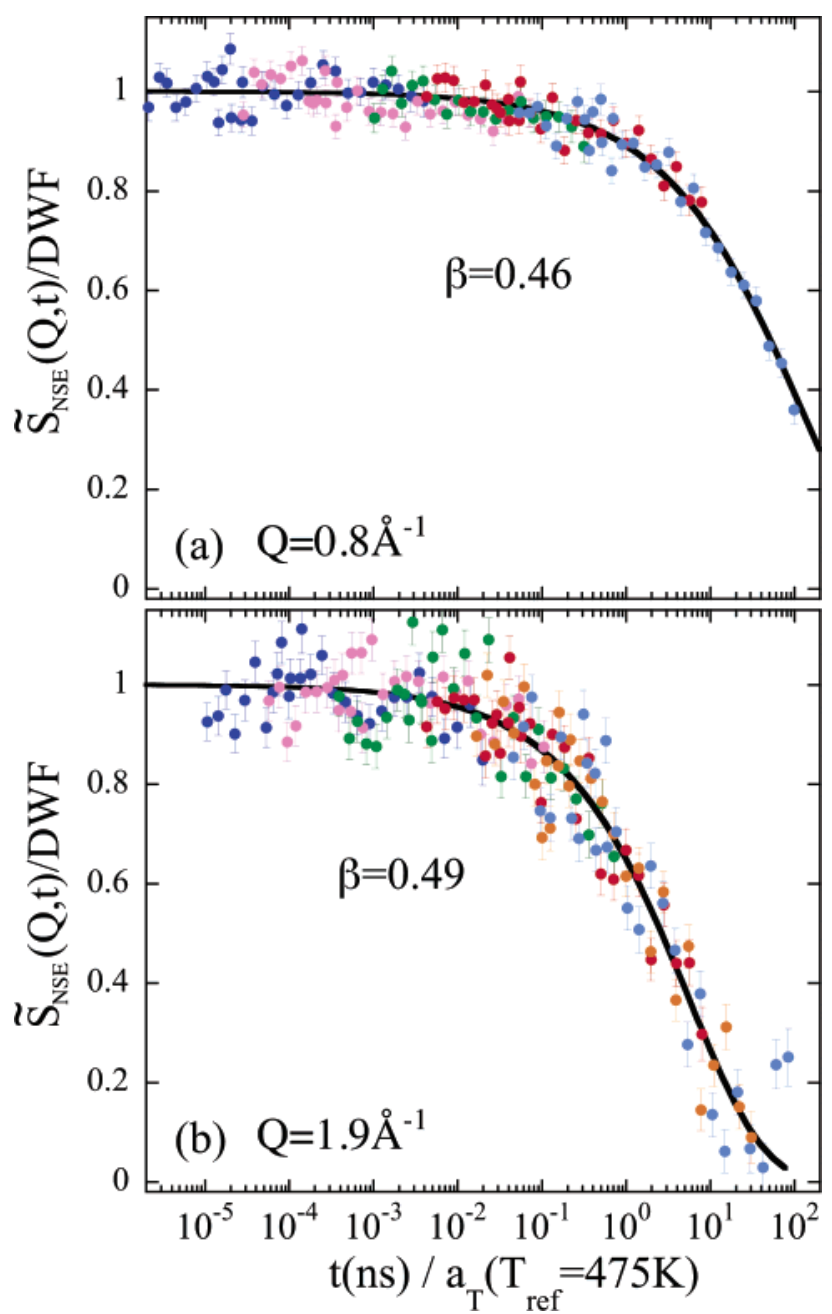

Figure 2. Master curves obtained from the NSE data normalized by the DWF with $475 \mathrm{~K}$ as reference temperature for (a) $Q=0.8 \AA^{-1}$ and (b) $Q=1.9 \AA^{-1}$. Symbols as shown in the inset of Figure 1a. The lines are KWW descriptions with $\beta=0.46$ in (a) and 0.49 in (b).

descriptions of the NSE data are achieved for all temperatures investigated. Removing the influence of the fast decay by renormalization with the obtained DWF, a direct comparison of the curves at both $Q$ values may be done for a given temperature. Figure 1c shows this comparison for $516 \mathrm{~K}$. There we clearly realize that the decay of the dynamic structure factor at its first maximum is significantly slower than at the second maximum. From the KWW fits of the master curves we found $\tau_{\mathrm{w}}\left(0.8 \AA^{-1}, T_{\mathrm{R}}\right)=115 \mathrm{~ns}$ and $\tau_{\mathrm{w}}\left(1.9 \AA^{-1}, T_{\mathrm{R}}\right)=5.6 \mathrm{~ns}$. Direct comparison of time scales corresponding to KWW functions with different shape parameters may be done through the average time, $\langle\tau\rangle$. This is the first moment of the distribution of simple exponential processes giving rise to the stretched exponential-at least from a mathematical point of view. For KWW functions

$$
\langle\tau\rangle=\frac{\Gamma\left(\frac{1}{\beta}\right)}{\beta} \tau_{\mathrm{w}}
$$

leading to $\langle\tau\rangle\left(0.8 \AA^{-1}, T_{\mathrm{R}}\right)=278 \mathrm{~ns}$ and $\langle\tau\rangle\left(1.9 \AA^{-1}, T_{\mathrm{R}}\right)=$ $11.6 \mathrm{~ns}$. Figure 3 shows the temperature dependence of the characteristic times obtained for the two $Q$ values in comparison with results reported for PMMA from dielectric spectroscopy ${ }^{13}$ and NMR. ${ }^{16}$ The NSE times have been reconstructed from the shift factors used to build the master curves, multiplying them by the corresponding values of $\langle\tau\rangle\left(Q, T_{\mathrm{R}}\right)$. 


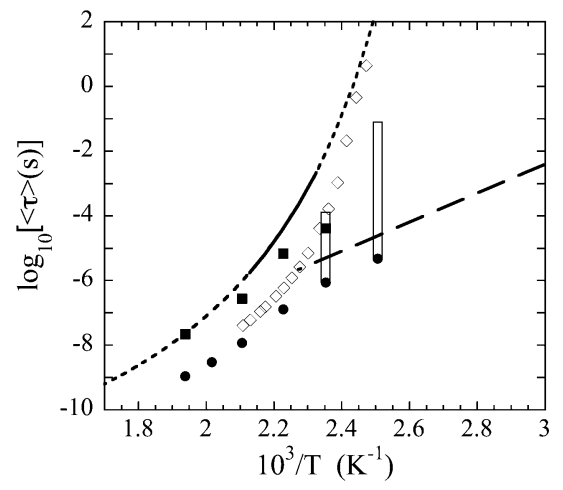

Figure 3. Temperature dependence of the average time characterizing the decay of the dynamic structure factor at $Q=0.8 \AA^{-1}$ (full squares) and $Q=1.9 \AA^{-1}$ (full circles). The data are compared with dielectric spectroscopy (diamonds) ${ }^{13}$ and NMR results. ${ }^{16}$ The solid part of the NMR line shows the experimentally accessed region, and the dotted lines correspond to the extrapolated results by the law given in ref 16 . The dashed line shows the $\beta$-relaxation characteristic time as determined from dielectric spectroscopy. ${ }^{13}$ The vertical bars link the NSE time scales obtained by considering $T$-constant (points) and $T$-dependent ${ }^{13}$ $\beta$ parameters.

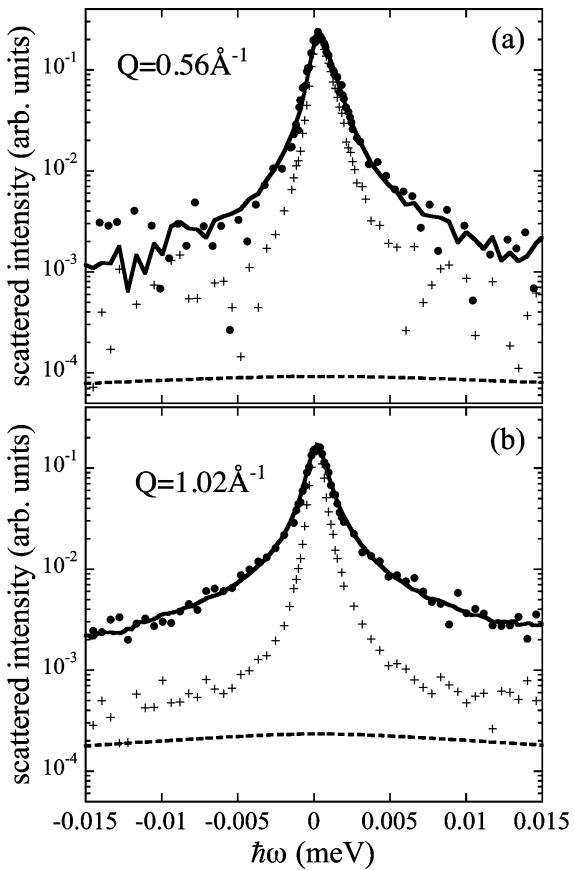

Figure 4. Backscattering spectra measured for the PMMA- $d_{3}$ sample at $518 \mathrm{~K}$ for $Q=0.56 \AA^{-1}$ (a) and $Q=1.02 \AA^{-1}$ (b). Solid lines are fitting curves obtained with a KWW function with $\beta=0.5$ and the fixed contribution giving account for the $\alpha$-methyl group rotation, which quasielastic part is displayed by the dotted line. The instrumental resolution is shown for comparison (crosses).

B. Backscattering. Because of the high value of $\sigma_{\text {inc }}^{\mathrm{H}}$ relative to the rest of the cross sections involved in the PMMA$d_{3}$ sample, the scattering from this system mainly reveals the self-correlation function of main-chain and $\alpha$-methyl group hydrogens. As examples, the BS spectra measured for $Q=0.56$ and $1.02 \AA^{-1}$ are shown in Figure 4 . The broadening, which is determined by the inverse of the characteristic time, increases with increasing $Q$ value, indicating some kind of diffusive-like behavior. In the case of simple diffusion, the dispersion law relating the characteristic time scale $\tau$ with $Q$ is given by $\tau \propto$ $Q^{-2}$. However, for protonated glass-forming polymers, much stronger $Q$ dependences are generally reported ${ }^{34,48,49}$ for the time scale associated with the $\alpha$-relaxation. Usually, in such cases, exponents close to 4 are found, which have been correlated with the non-Debye behavior of the intermediate scattering function. ${ }^{48}$ It is well established that in the BS window the signal produced by hydrogen motions in the structural relaxation regime is well described in terms of the Fourier transform of a KWW function in the time domain; i.e., $S_{\mathrm{s}}(Q, t)$ takes an analogous form of eq 6:

$$
S_{\mathrm{s}}(Q, t)=A(Q) \exp \left[-\left(\frac{t}{\tau_{\mathrm{w}}}\right)^{\beta}\right]
$$

We note that in the incoherent case the amplitude $A(Q)$ (also known as Lamb-Mössbauer factor) reveals the mean-square displacement associated with the fast dynamics, $\left\langle u^{2}\right\rangle$, through $A(Q)=\exp \left[-Q^{2}\left\langle u^{2}\right\rangle / 3\right]$. It is important to emphasize that the Debye-Waller factor, which refers to coherent scattering, i.e., pair correlations, is also $Q$-dependent. However, it does not follow the $Q^{2}$ dependence displayed by $A(Q)$ in the exponential and usually is modulated by $S(Q) . .^{50}$

We have assumed the description of eq 8 for the incoherent scattering function of the segmental motion of main-chain and $\alpha$-methyl hydrogens. In addition, other contributions have to be considered to account for the measured spectra. First, we note that in our PMMA- $d_{3}$ sample the hydrogens in the $\alpha$-methyl group contribute with an additional motion to the segmental dynamics: the methyl group rotation. A recent characterization of this process in a blend of PMMA with poly(ethylene oxide) $(\mathrm{PEO})^{8}$ delivers an Arrhenius law with a prefactor of $65.52 \mathrm{meV}$ and an activation energy of $290 \mathrm{meV}$ for the characteristic rate $\Gamma_{0}$. On this basis, the estimation for $\Gamma_{0}$ at $518 \mathrm{~K}$ is $99 \mu \mathrm{eV}$; i.e., the rotation is very fast. Moreover, a distribution of rates with a width of 0.38 decades is expected. In fact, the quasielastic contribution of this process to the BS window is very weak and nearly flat (see Figure 4). The expected contribution of this motion to the spectrum was thus taken into account in addition to the KWW function to model the total scattering function.

Second, the measured spectra also contain a coherent contribution which will be nonnegligible close to the maximum of the corresponding partial static structure factor (up to $25 \%$ at $1.3 \AA^{-1}$; see Figure 1 in ref 21). The characterization of the dynamics of such contribution is very complicated. In PMMA$d_{3}$, the coherent scattering reflects to a large extent correlations relating to the ester side groups. ${ }^{21}$ As we will see later, the characteristic times of collective motions involving these groups are similar in the high- $Q$ regime to those corresponding to incoherent segmental dynamics. Thus, the same KWW function accounting for the segmental motion of hydrogens was used to describe the coherent contribution close to the peak. In the other $Q$-regions, this contribution can be safely neglected.

In the fitting procedure the $\beta$ value was fixed to 0.5 , which is close to the values obtained for coherent scattering at the two maxima and, as we will see below, is the stretching parameter fitting the MD simulations results. Figure 4 shows the good quality of the descriptions obtained with this model function convoluted with the instrumental resolution. The characteristic times deduced from the fit are shown in Figure 5a.

\section{Validation of Simulations}

As the second in the series, this paper deals with the dynamical features of PMMA in the region close and above the glass transition. For the interpretation of the dynamic data the comparison with molecular dynamic simulation which have been performed in parallel is crucial. Therefore, we will start with a validation of these MD simulations. As shown in ref 21, 


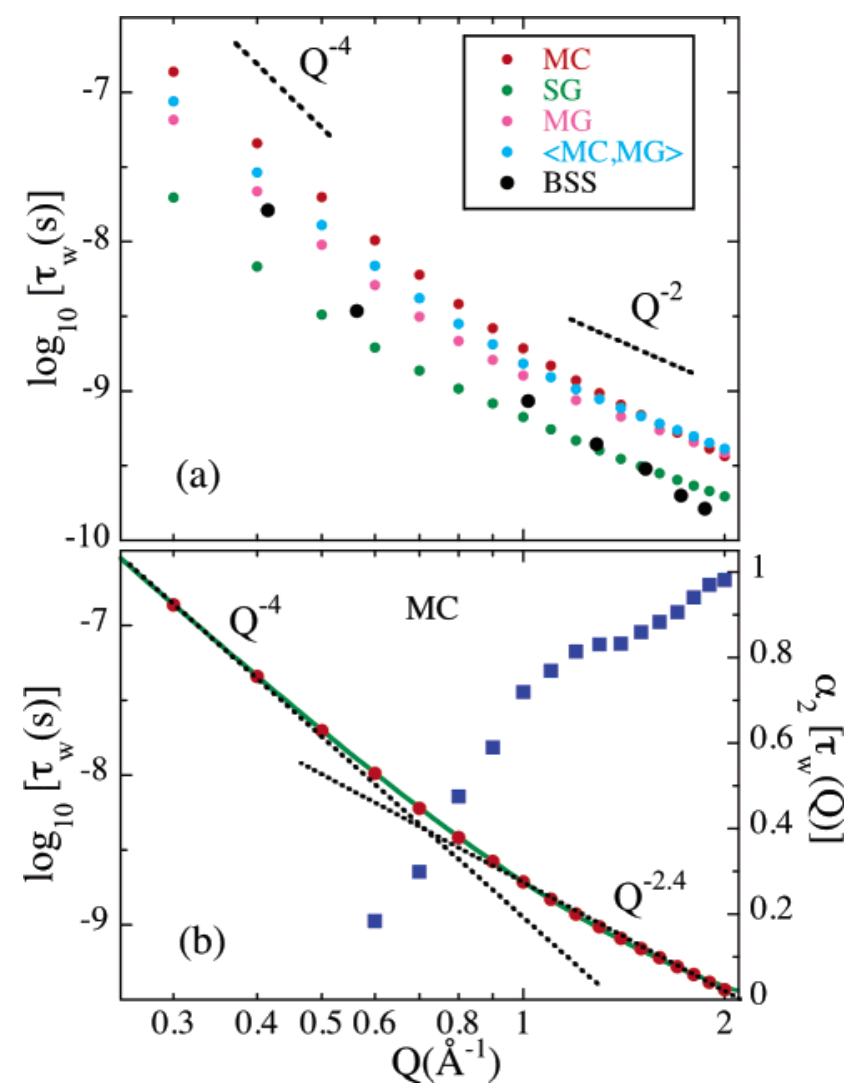

Figure 5. (a) Momentum transfer dependence of the KWW characteristic times obtained by BS at $518 \mathrm{~K}$ (black) and from the simulations at $580 \mathrm{~K}$ for the hydrogens in the ester methyl group (green), in the main chain (red), and in the $\alpha$-methyl group (pink) and when considering the two later together (blue). (b) For main-chain hydrogens, the value of the non-Gaussian parameter evaluated at the characteristic time for each momentum transfer is plotted together with the characteristic times. The green line is a description in terms of the anomalous jump diffusion model with $\zeta_{0}=0.6 \AA$. The dashed lines show the indicated power laws.

the simulations very well reproduce the structural features of PMMA. This is not only true for the coherent structure factor obtained from a fully deuterated material, but also for the five differently weighted partial structural factors which may be observed experimentally in labeling with deuterium at different positions of the molecule. The comparison with $\mathrm{X}$-rays results reported in the literature ${ }^{35-37}$ is successful as well. ${ }^{21}$ Thus, the simulations lead to an overall good agreement with the experimental partial static structure factors.

After validating the structure, we also have to compare simulations and experiments relating to the dynamics. A direct comparison of NSE and simulation results is not possible without considering slight amplitude corrections for the experimental data. ${ }^{38,39}$ These arise from a deficient normalization of the NSE signal. As a result of the polarization analysis after the scattering process, a NSE spectrometer offers only a limited band-pass to the scattered neutrons. ${ }^{26}$ Inelastically scattered neutrons with energies higher than those allowed by the bandpass (about $2 \mathrm{meV}$ in the case of IN11) are not transmitted through the instrument and do not participate in the normalization event. As already mentioned, this implies that the value experimentally obtained for the static limit of $S_{\text {coh }}(Q, t)$ is not exactly $S(Q)$. Therefore, the measured NSE signal differs from the properly normalized scattering function in a factor $A_{\mathrm{BP}}(Q)$, which translates into an effectively smaller DWF. Following the same procedure as in ref 39, we have corrected the NSE amplitudes by using the simulation results, where the uncertain-

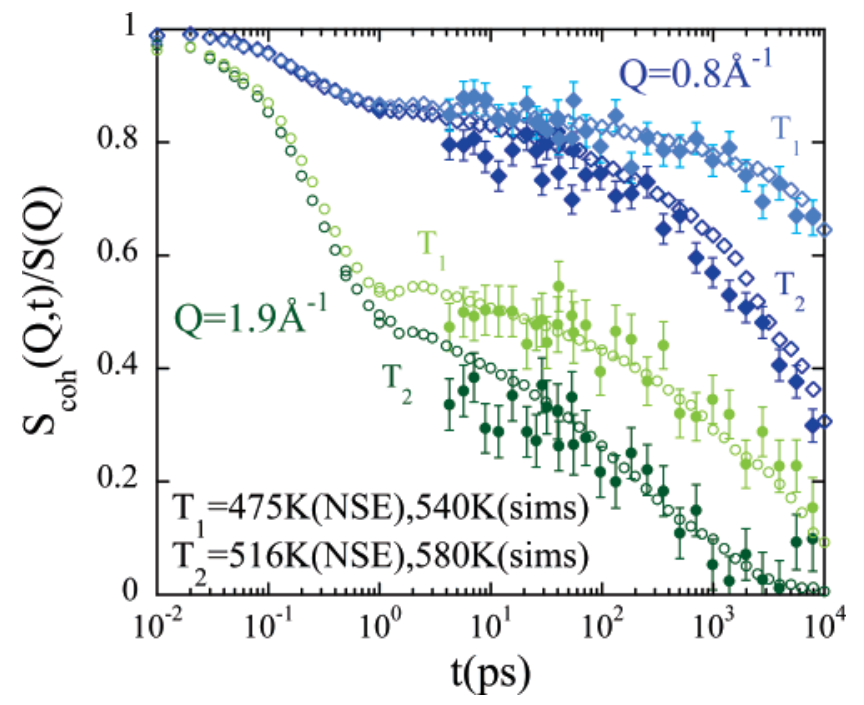

Figure 6. Dynamic structure factor of PMMA: simulation results (empty symbols) and IN11 results (full symbols) for $Q=0.8 \AA^{-1}$ (rhombs) and $Q=1.9 \AA^{-1}$ (circles). The amplitudes of the experimental data have been corrected for the band-pass of the spectrometer using the MD simulations results at $t=0.2 \mathrm{ps}$.

ties involved in the calculation of the static value of $S_{\mathrm{coh}}(Q, t)$ are very small. The factor $A_{\mathrm{BP}}$ may be related to the value of the dynamic structure factor in an "effective band-pass time" $t_{\mathrm{BP}}, S_{\mathrm{coh}}\left(Q, t_{\mathrm{BP}}\right)$. This time should be of the order of the inverse of the band-pass frequency, $t_{\mathrm{BP}} \approx 0.3 \mathrm{ps}$. We have found a reasonable agreement assuming $t_{\mathrm{BP}} \approx 0.2 \mathrm{ps}$, similar to what it is expected and what it was found in ref 39 for poly(vinyl ethylene). For the highest temperature $(516 \mathrm{~K})$, the such corrected spectra are compared with the simulation results at $580 \mathrm{~K}$ in Figure 6. There, the simulation data have been calculated by means of eq 5 and then normalizing the results by $S(Q)$. We find a very good agreement between both sets of data. This concerns not only the decay of the relaxation function which is observed at times above 2 ps but also the initial decay relating to fast dynamics such as librations and the Boson peak in glasses. Thus, allowing the same shift in temperature (65 $\mathrm{K}$ ), we find, as for the validation of the static data, that the dynamics is also well reproduced by our simulated cell. To show that the simulations are consistent with the experimental results maintaining this shift, we have also run a simulation at $540 \mathrm{~K}$, i.e., $40 \mathrm{~K}$ below the previous simulation. As can be seen in Figure 6, the experimental data obtained for PMMA at $475 \mathrm{~K}$ and corrected as explained above perfectly agree with these results. Thus, the required shift is similar for both sets of data. We note that the simulations carried out by Soldera et al. ${ }^{18}$ on the same system lead to similar time scales as in our case, implying the need of the same shift in temperature to match the experimental results. Though smaller (about 30-40 K), some temperature shift had also to be invoked for polyisoprene ${ }^{38}$ and $o$-terphenyl. ${ }^{40}$ Apart from slight differences in the microstructure or in the density, a possible source for this discrepancy could be that the force field used in the simulations was not perfectly calibrated. For the case of polybutadiene, it has been shown by Smith et al. ${ }^{41}$ that the time scale of the $\alpha$-relaxation resulting from the MD simulations is quite sensitive to slight changes of the local intrachain potentials. In this direction, the comparison with experiments as done here could be used to refine the rotational potentials of the force field for PMMA.

There is still another observation that supports the validation of our cell from a dynamic point of view. As we will show in the next section, we also achieve good agreement for the 
characteristic times for the hydrogen self-motion as obtained from BS at $518 \mathrm{~K}$ and from the simulations at $580 \mathrm{~K}$. Thus, allowing the mentioned temperature shift, both correlation functions (pair and self) are well reproduced, a result which is not trivial.

\section{Discussion}

A. Hydrogen-Weighted Self-Terms: Incoherent Scattering. To a large extent, the BS measurements on the PMMA- $d_{3}$ sample reveal the self-motions of the hydrogens at the main chain and in the $\alpha$-methyl group. The simulations allow an inspection of the self-correlation functions for each kind of atom in the sample. For $Q=1 \AA^{-1}$, Figure 7 shows these functions in $Q$-space $S_{\mathrm{s}}^{\alpha}(Q, t)$ (eqs 2 and 3). They are calculated for the three types of hydrogens in the sample [ $\alpha$ : main-chain hydrogen $\left(\mathrm{H}^{\mathrm{MC}}\right), \alpha$-methyl group hydrogen $\left(\mathrm{H}^{\mathrm{MG}}\right)$, ester side group hydrogen $\left(\mathrm{H}^{\mathrm{SG}}\right)$ ]. For $\mathrm{H}^{\mathrm{MC}}$ and $\mathrm{H}^{\mathrm{SG}}$ we observe a qualitatively similar behavior: after a fast decay until $\approx 1 \mathrm{ps,} \mathrm{a} \mathrm{second} \mathrm{slow}$ regime leads to the total decay of the correlations. In a first approximation (we will see later that for $\mathrm{H}^{\mathrm{SG}}$ the situation is more complex), these two steps can be attributed to the fast dynamical processes and the $\alpha$-relaxation, respectively. The $\mathrm{H}^{\mathrm{MG}}$ present in addition a third intermediate regime. This can be identified as due to the $\alpha$-methyl group rotations. In fact, rotations are also active in the ester methyl groups. However, the associated time scale there is so fast that this process appears just as taking part of the fast dynamics.

Our main focus is to characterize the dynamics at times larger than the picosecond scale. There, the contributions of the fast processes can be effectively parametrized with an amplitude for the rest of the decay, $A(Q)$. Then, for the main-chain hydrogens as well as for the ester methyl group hydrogens-at least in a first approach-the self-correlation function can be well described by eq 8 . From the corresponding fits, an approximately $Q$-independent value of $\beta=0.5$ is found for both kinds of atoms. By fixing the stretching parameter to this value the good descriptions shown in Figure 7 are obtained. In the case of $\mathrm{H}^{\mathrm{MG}}$ both the methyl group rotation and the segmental motions have to be taken into account. As already shown for PMMA in a blend with $\mathrm{PEO},{ }^{8}$ a good approximation is to consider that both kinds of motions take place simultaneously and independently. Then the total intermediate scattering function is given by the product of the respective scattering functions:

$$
S_{\mathrm{s}}^{\mathrm{total}}(Q, t)=A(Q) S_{\mathrm{s}}^{\mathrm{I}}(Q, t) S_{\mathrm{s}}^{\mathrm{II}}(Q, t)
$$

Here $S_{\mathrm{s}}^{\mathrm{I}}(Q, t)$ is the KWW function corresponding to the $\alpha$-relaxation. For a localized motion-like the rotation of the $\mathrm{MG}$-the scattering function $S_{\mathrm{s}}^{\mathrm{II}}(Q, t)$ assumes the form

$$
S_{\mathrm{S}}^{\mathrm{II}}(Q, t)=\mathrm{EISF}+(1-\mathrm{EISF}) \phi(t)
$$

The elastic incoherent structure factor EISF carries the information about the geometry of the particular motion involved. In this particular case-a rotational jump between three equivalent positions-it is given by

$$
\mathrm{EISF}_{\mathrm{MG}}=\frac{1}{3}\left(1+2 \frac{\sin \left(Q r_{\mathrm{HH}}\right)}{Q r_{\mathrm{HH}}}\right)
$$

( $r_{\mathrm{HH}}=1.78 \AA$ : distance between the hydrogens). The timedependent function $\phi(t)$ in eq 10 is normalized (decays between 1 and 0 ) and depends on the characteristic rate $\Gamma$ of the localized

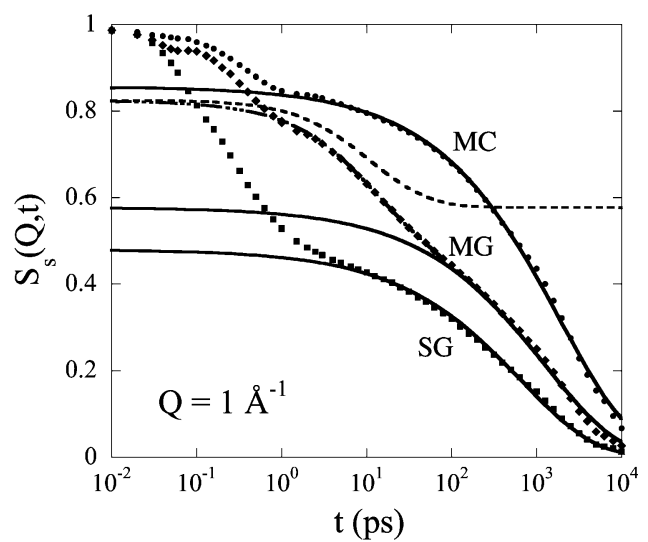

Figure 7. Self-correlation function obtained from the simulations at $Q=1 \AA^{-1}$ for the hydrogens at the main chain (circles), in the $\alpha$-methyl group (rhombs), and in the ester methyl group (squares). Solid lines are $\mathrm{KWW}$ functions with prefactors $A(Q)$ giving account for the decay below $\approx 1$ ps $(\mathrm{MC}, \mathrm{SG})$ and with prefactor $A(Q) \mathrm{EISF}_{\mathrm{MG}}(\mathrm{MG})$. In the latter case, the total description above 1 ps is given by the dasheddotted line, and the contribution of the methyl group rotation [multiplied by $A(Q)]$ is depicted by the dotted line.

motion. Because of the disorder in the system, different environments leading to distribution of rates are present, and consequently $\phi(t)$ is not a simple exponential decay. For methyl group rotations a Gaussian distribution function of $\Gamma$ is usually assumed:

$$
\phi_{\mathrm{MG}}(t)=\int_{+\infty}^{-\infty} \frac{1}{\sqrt{2 \pi} \sigma} \mathrm{e}^{\left[-\left(\log \Gamma-\log \Gamma_{0}\right)^{2} / 2 \sigma^{2}\right]} \mathrm{e}^{-\Gamma t} \mathrm{~d}(\log \Gamma)
$$

In the range of applicability of this model function (after the fast microscopic decay) the achieved description of the MD simulation results for $\mathrm{H}^{\mathrm{MG}}$ is excellent, as can be appreciated in Figure 7. From the fit, a mean value of $\Gamma_{0}=48.2 \mu \mathrm{eV}$ and a width $\sigma$ of 0.40 decades are found for the frequency distributions function. Compared with the experimentally expected values at the equivalent temperature $(99 \mu \mathrm{eV}$ and 0.38 decades, see section IV.B), we find just the same width and a rate within a factor of 2 , i.e., a rather good agreement. The contribution of this motion to the decay of the correlations [affected by the amplitude factor $A(Q)$ ] is separately shown in Figure 7 (dotted line). The $\alpha$-relaxation contribution [multiplied by the asymptotic value of the methyl group rotation for longer times, $A(Q)$ EISF $\left._{\mathrm{MG}}\right]$ is displayed as the solid line. As expected, it describes the long-time decay of the correlations. The stretching parameter was again fixed to $\beta=0.5$.

The $Q$-dependent characteristic times deduced for segmental motion for the three kinds of atoms, together with the experimental results (black dots), are displayed in Figure 5awe remind that the BS data have also been fitted with the same functional form $(\beta=0.5)$. For a direct comparison of the results, we have calculated the weighted sum of the main-chain and the $\alpha$-methyl group contributions (full blue points) which together correspond to the experimental observation (apart from small coherent contributions). We note that the BS points agree within a factor of 2-3 with the simulations - an overall very respectable result that supports again the validation of the cell.

The $Q$ dependence of the experimental results appears to show an increasing slope in going from high to lower $Q$. This behavior can be quantitatively read off from the computer simulation. Concentrating first on the main chain (see Figure 5 b), we realize that the $Q$ dispersion changes from an exponent of 2.4 at high $Q$ to an exponent close to 4 (a fit delivers 3.65) at low $Q$. A similar behavior has been observed in several instances for other 
systems ${ }^{38,39,42-46}$ and could be interpreted in terms of a crossover from heterogeneous dynamics at local scales to Gaussian relaxation at larger scales. ${ }^{38,47}$ As shown in previous publications, ${ }^{48,49}$ such a Gaussian process leads to a relation between the stretching exponent $\beta$ and the $Q$ dispersion of the characteristic times $\tau_{\mathrm{w}} \propto Q^{-2 / \beta}$. While at low $Q$ this relationship is rather well fulfilled in PMMA, around $Q \approx 0.8 \AA^{-1}$ severe deviations appear. To further investigate this observation, we have calculated the non-Gaussianity parameter for the dynamics of the main-chain hydrogens. The non-Gaussian parameter $\alpha_{2}(t)$ is given by a combination of moments of the self-correlation function

$$
\alpha_{2}(t)=\frac{3}{5} \frac{\left\langle r^{4}(t)\right\rangle}{\left\langle r^{2}(t)\right\rangle^{2}}-1
$$

Here $\left\langle r^{2 n}\right\rangle$ is the $2 n$ moment of the radial self-correlation function $G_{\mathrm{s}}(r, t) . \alpha_{2}(t)$ indicates the leading order deviation from Gaussianity and vanishes for a Gaussian process. In Figure $5 \mathrm{~b}$ we present the non-Gaussianity parameter $\alpha_{2}$ taken at the characteristic relaxation time $\tau_{\mathrm{w}}(Q)$. We realize that, at this time and at high $Q, \alpha_{2}$ is large, indicating strongly non-Gaussian dynamics. At the crossover point $\left(Q \approx 0.7 \AA^{-1}\right) \alpha_{2}$ is about 0.4 , with a tendency to strongly decrease toward lower $Q$ or longer times. The asymptotic slope of 4 -required for a Gaussian process with $\beta=0.5-$ is hardly reached within the $Q$ window of the simulation and the experiment. This may result from the fact that only around $Q \approx 0.5 \AA^{-1}$ the non-Gaussianity parameter becomes negligible. Similar observations were reported for polyisoprene, ${ }^{38}$ where this crossover takes place around $Q \approx 1.3 \AA^{-1}$. Neutron scattering studies and/or simulations on different polymers have also revealed deviations from Gaussian behavior in polybutadiene ${ }^{44}$ and polyisobutylene ${ }^{42,43}$ at $Q \approx 1 \AA^{-1}$ and in poly(vinyl ethylene), ${ }^{39}$ poly(vinyl acetate), ${ }^{45}$ poly(ethylene propylene), ${ }^{46}$ and head-to-head-polypropylene ${ }^{46}$ at about $Q \approx 0.65 \AA^{-1}$. This means that this crossover seems to be a universal feature for the atomic motions in the $\alpha$-relaxation regime. A possible interpretation of this finding was proposed in ref 38, namely, a distribution of jumps underlying the anomalous diffusive motion of protons in the $\alpha$-process. Assuming the superposition of small discrete jumps of different lengths $/$ with a most probable value $/ 0$, the resulting characteristic time is given by

$$
\tau_{\mathrm{w}}=\tau_{0}\left[1+\frac{1}{Q^{2} l_{0}^{2}}\right]^{1 / \beta}
$$

which in the asymptotic low- $Q$ limit yields the Gaussian law $\tau_{\mathrm{w}} \propto Q^{-2 / \beta}$. As can be appreciated in Figure $5 \mathrm{~b}$, the dispersion of the characteristic time for main-chain hydrogens in PMMA is perfectly described in this framework. A value of $l_{0}=0.6 \AA$ is deduced, larger than that found for polyisoprene $\left(l_{0}=0.4\right.$ $\AA) .{ }^{38}$ Thus, compared with that case, the bulky monomer of PMMA seemingly needs larger scales in order to escape the heterogeneities imposed by structural constraints.

Compared to the main-chain characteristic times, those of the side groups are significantly faster. In particular, that for the ester side group differs by about a factor of 2 at high $Q$, while at low $Q$ this difference increases to nearly an order of magnitude (factor 7-8). This increasing difference relates to the much stronger dispersion of the main-chain dynamics compared to that of the side group. While the result at high $Q$ could be easily rationalized, the order of magnitude difference at low $Q$, where we look on large scales, is somewhat surprising.



Figure 8. Additional contribution to the dynamics of the ester methyl group hydrogens with respect to the main-chain segmental motion at the $Q$ values indicated. The solid lines are fits to eq 10 with free EISF and a KWW function with $\beta=0.5$ and $\tau_{\mathrm{w}}=3.8 \mathrm{~ns}$ for $\phi(t)$. A prefactor $A(Q)$ gives account for the fast decay below $1 \mathrm{ps}$.

In the following we will try to explain these observed differences in the characteristic times.

Side groups as the ester group in PMMA may show additional dynamical processes superimposed on the segmental motion that is shared with the backbone atoms. For example, for this group $\pi$-flips have been reported from NMR studies. ${ }^{11}$ In such a case, we cannot expect the decay of the self-correlation function to be the same as that observed for the main-chain atoms. We thus may assume the occurrence of some additional motion for $H^{\mathrm{SG}}$ that, in a first approach, could be considered statistically independent from the main-chain dynamics. As we have seen, the total correlation function then would be expressed by eq 9 . Now, function I would correspond to the segmental motionas determined from the slow decay of the main-chain hydrogens results - and function II would account for the "additional" motion of the ester hydrogens with respect to the main-chain dynamics. We can easily deduce $S_{\mathrm{s}}^{\text {add }}(Q, t)$ by simply dividing $S_{\mathrm{s}}^{\mathrm{H}^{\mathrm{SG}}}(Q, t)$ by $S^{\mathrm{I}}(Q, t)=S^{\mathrm{H}^{\mathrm{MC}}}(Q, t) / A_{\mathrm{H}^{\mathrm{MC}}}(Q)$.

The result is displayed in Figure 8 . We note that this procedure applies for $t \gtrsim 1 \mathrm{ps}$, and the resulting function still contains the decay in the fast regime on the short-time side of the "additional" process contribution. On the other hand, we can appreciate that this additional process is by no means negligible, even at $Q$ values as low as $0.4 \AA^{-1}$. For that low $Q$ value, this motion leads to the decay of the self-correlation to almost half of its initial value at $1 \mathrm{ps}$. This indicates that the spatial extent of this dynamical process has to be large. To estimate its characteristic length scale, we apply the generic description of eq 10. As a first approximation, we consider a $Q$-independent dynamic function $\phi(t)$ using a KWW-like functional form with $\beta=0.5$ and $\tau_{\mathrm{w}}=3.8 \mathrm{~ns}$ that delivers a rather good description of the data (see Figure 8). The $Q$-dependent values obtained for the corresponding EISF are displayed in Figure 9. They have been compared with the predictions of several models for localized motions: (i) isotropic rotational diffusion on a sphere of radius $R$ and (ii) jumps among $N$ equivalent sites equally spaced on a circle of radius $R$ for different values of $N .^{24}$ These theoretical functions are plotted versus the dimensionless variable $Q R$. We observe a reasonable agreement between our results and a model considering a large number of jumps on a circle (i.e., some sort of continuous diffusion) for a value of $R \approx 4 \AA$. It is interesting to note that this distance is very close to that between ester methyl group hydrogens and the corresponding main-chain carbon. Thus, we may interpret the additional motion as some kind of rotation of 




Figure 9. Elastic incoherent structure factor obtained for the additional motion performed by the ester methyl group hydrogens with respect to the main-chain motion (blue squares) at times longer than $1 \mathrm{ps}$. The predicted functions for some confined motions are shown for comparison: jumps among $N$ equivalent sites equally spaced on a circle and isotropic rotational diffusion on a sphere. The functions are plotted against $Q R$, where $R$ is the radius of the circle and the sphere, respectively. For the simulation data the value $R=4 \AA$ has been fixed.

the ester methyl group around the main chain. A full characterization of this motion is beyond the scope of this paper and will be addressed in a future work involving BS measurements on the sample PMMA- $d_{5}$ (hydrogens only at the ester methyl group). Nevertheless, it is important to emphasize that the spatial scale of the rotational motion we have identified well above $T_{\mathrm{g}}$ is well beyond what would be expected for a simple $\pi$-flip ( $N$ $=2, R \approx 2 \AA$ ) which was observed in the neighborhood of $T_{\mathrm{g}}$.

Finally, we note that the dynamics for PMMA within the spatial scale of BS is very heterogeneous: from the fast rotations of the methyl groups to the much slower rotations and translations of the backbone. Therefore, the effective $Q$ dependence of the scattering from a fully protonated sample, without a distinction of the different types of dynamics may lead to any power-law exponent. This might be the reason why from a BS study of such a sample at similar temperatures than those here investigated García Sakai et al. ${ }^{19}$ obtained a $Q$ dependence for the characteristic time close to $Q^{-2}$ in the whole $Q$ range accessed $\left(0.6 \lesssim Q \lesssim 1.8 \AA^{-1}\right)$.

B. Collective Dynamics. After having validated the simulation results on the collective dynamics with the NSE data (Figure 6), we now focus on the discussion of the simulation data to unravel more details of these collective dynamics. We will concentrate on the partial structure factors related to the molecular building blocks as was done in the discussion of the PMMA structure. ${ }^{21}$ As explained in ref 21, we distinguish three different groupings (see inset in Figure 10): the main chain (MC), the ester side group (SG), and the $\alpha$-methyl group (MG). Including all interference terms this leads to six different partial structure functions $a_{G / G^{\prime}}(Q, t)$ with $G, G^{\prime}$ : MC, MG, SG. Properly weighted by the corresponding scattering lengths, these functions deliver the contributions to the coherent scattering which is accessed experimentally. The very similar values of the neutron scattering lengths of carbons, deuterons, and oxygens lead to the direct observation of the structure factor in fully deuterated PMMA (all atoms are almost equally weighted). In the static

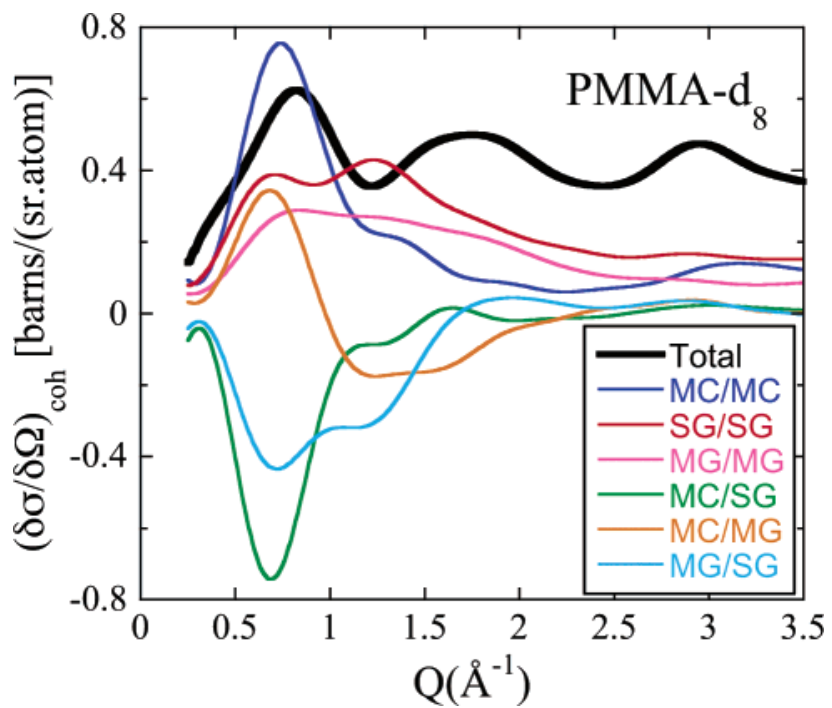

Figure 10. Static structure factor corresponding to the fully deuterated sample PMMA- $d_{8}$ (black curve). The different contributions from the partial correlation functions corresponding to the atoms within the molecular groups considered are also shown.

case, this corresponds to the static structure factor $S(Q)$. This is shown in Figure 10 together with its different partial contributions from the distinguished atomic groups. The main conclusions on the structural results were that: (i) PMMA is a strongly locally ordered glassy system: the side group areas, and there mainly the ester methyl groups, are very strongly anticorrelated to the main chain. This structure appears to be a precursor effect to the invoked nanophase separation in higher order Pn-MA's. (ii) The main-chain correlations are very strong-the $\mathrm{MC} / \mathrm{MC}$ peak is about 6 times higher than the high- $Q$ average. (iii) The first peak of the structure factor, though resulting from a superposition of several partial structure functions, represents the overall periodicity of the system with an average main-chain distance of about $8.6 \AA$. On the other hand, the higher order peaks in the overall structure factor are not easily related to any particular feature of the partial factors and originate from a superposition of positive and negative contributions of trailingoff partial structure functions. We will need Figure 10 when we discuss particular features of the collective dynamics of PMMA. In the case of correlated dynamics of atomic pairs, coherent scattering evidences such collectivity through the $Q$ dependence of the inelastic contribution. If the motions of the two atoms considered are uncorrelated, the inelastic contribution to the dynamic structure factor just coincides with the inelastic self-correlation counterpart. Therefore, the direct comparison of the time scales obtained from both coherent (pair) and incoherent (self) scattering provides information on the degree of collectivity of the motions displayed by the different molecular groups in the polymer.

We commence with the region around the first peak representing the basic periodicity of the PMMA structure. Figure 11a displays the simulated dynamics of the different partial structure functions at this $Q$ point. We realize that these functions can be grouped in two different classes according to whether they relate to the main chain or not. Those relating to the main chain exhibit a very large amplitude (DWF)-the curves nearly start at 1 -and display a $50 \%$ decay within the window of observation. On the other hand, those relating to side-group (both SG and MG) dynamics (i) display a significantly smaller amplitude and furthermore (ii) decay to a much larger extent. The clearly smaller amplitude relates to librational degrees of freedom which are not available for the main chain, 


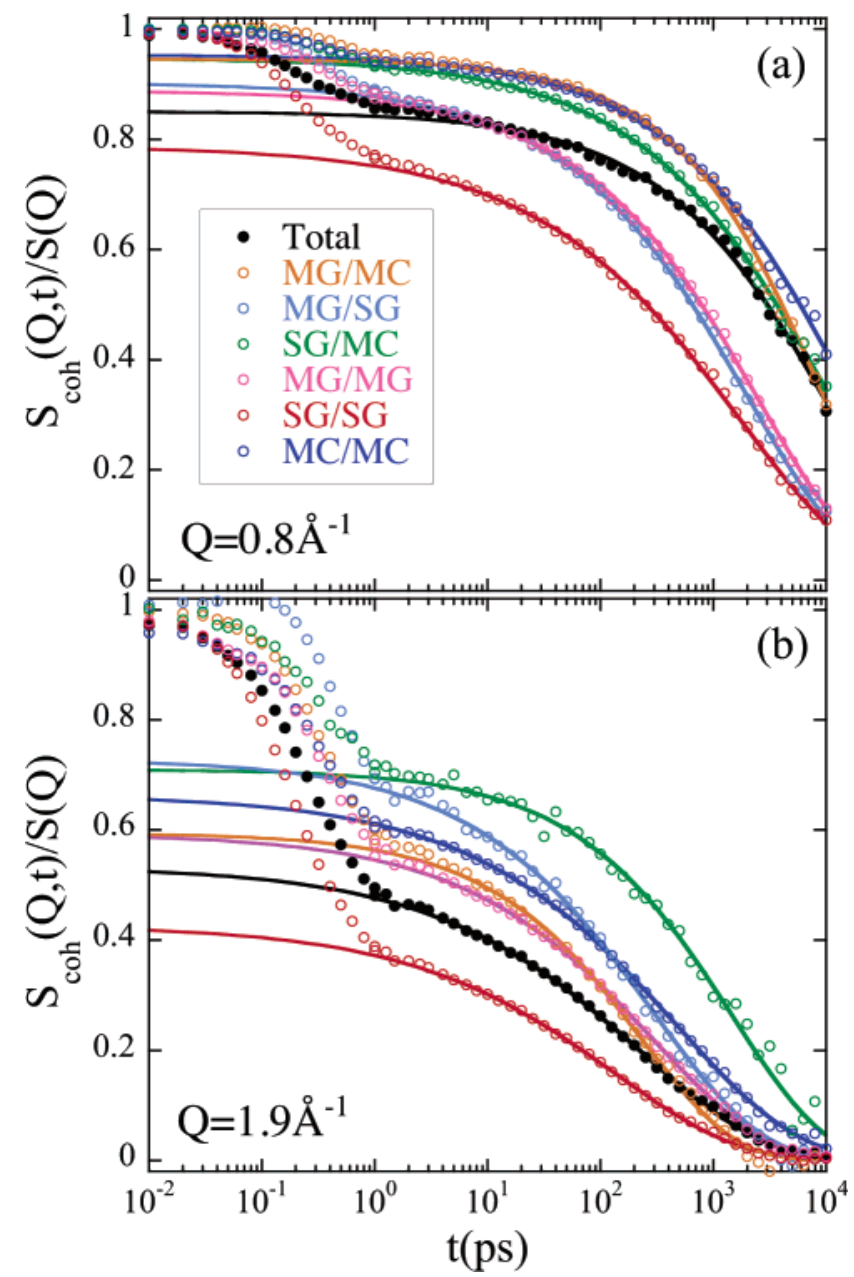

Figure 11. Normalized partial dynamic structure factors corresponding to the different atomic groups considered [see the inset in (a) for the color code]. The total dynamic structure factor is shown in black. The lines are KWW descriptions for $t \geq 4$ ps. (a) $Q=0.8 \AA^{-1}$; (b) $Q=$ $1.9 \AA^{-1}$.

Table 1. Characteristic Times and Shape Parameters Obtained for the Different Partial Dynamic Structure Factors and Characteristic Times of the Incoherent Scattering Function $(\boldsymbol{\beta}=\mathbf{0 . 5})$

\begin{tabular}{clllllll}
\hline$Q\left(\AA^{-1}\right)$ & subgroup & DWF & $\log \tau_{\mathrm{w}}(\mathrm{s})$ & $\beta$ & $\log \langle\tau(\mathrm{s})\rangle$ & $A(Q)$ & $\log \langle\tau\rangle^{\text {self }}(\mathrm{s})$ \\
\hline 0.8 & MC/MC & 0.953 & -7.831 & 0.47 & -7.489 & 0.927 & -8.036 \\
0.8 & MG/MG & 0.888 & -8.598 & 0.47 & -8.255 & 0.916 & $-8.297^{a}$ \\
0.8 & SG/SG & 0.787 & -8.756 & 0.41 & -8.264 & 0.759 & -8.535 \\
0.8 & MC/SG & 0.947 & -8.006 & 0.45 & -7.612 & & \\
0.8 & MG/MC & 0.946 & -8.054 & 0.58 & -7.856 & & \\
0.8 & MG/SG & 0.903 & -8.693 & 0.46 & -8.320 & & \\
1.9 & MC/MC & 0.663 & -9.318 & 0.40 & -8.797 & 0.631 & -9.057 \\
1.9 & MG/MG & 0.593 & -9.525 & 0.44 & -9.109 & 0.726 & $-9.199^{a}$ \\
1.9 & SG/SG & 0.427 & -9.860 & 0.40 & -9.343 & 0.307 & -9.344 \\
1.9 & MC/SG & 0.710 & -8.826 & 0.53 & -8.570 & & \\
1.9 & MG/MC & 0.594 & -9.627 & 0.53 & -9.378 & & \\
1.9 & MG/SG & 0.728 & -9.526 & 0.46 & -9.152 & & \\
${ }^{a}$ Only the carbon atom. & & & & &
\end{tabular}

while the stronger decay indicates larger mobility of the side groups, in particular the ester methyl group. Table 1 displays the DWF, the characteristic times, and the associated stretching parameters obtained from fits of eq 6 to the long-time decays of these functions. The corresponding average values (eq 7) are also shown in Figure 12a. The characteristic times are spread over more than 1 order of magnitude in time; e.g., for the SG/ SG correlation function, it is about 10 times faster than the $\mathrm{MC} /$ MC counterpart. The table and the figure also include results for the self-dynamics of the different molecular units irrespective

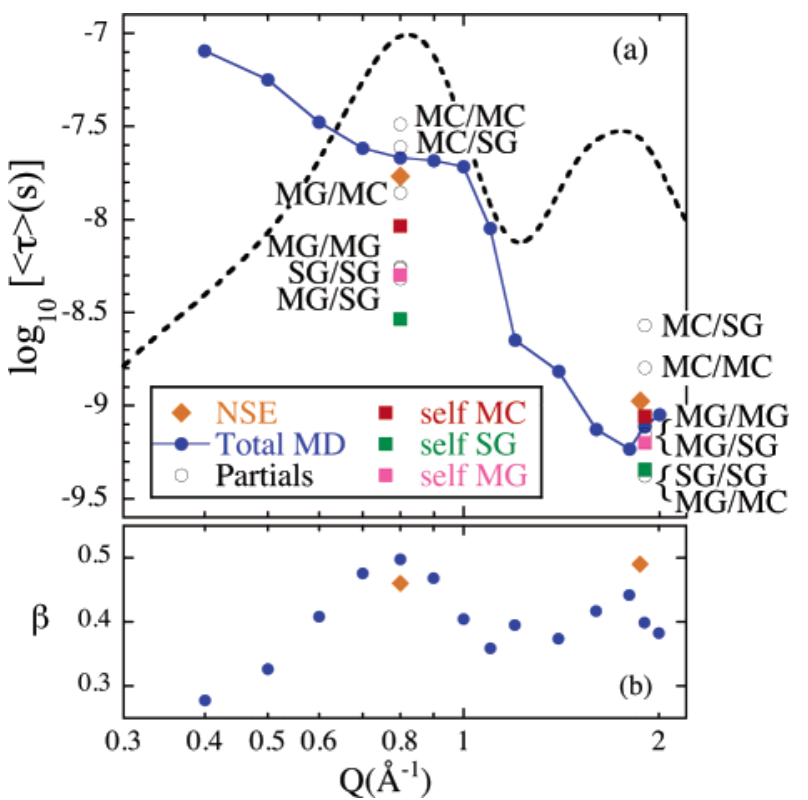

Figure 12. Momentum transfer dependence of (a) the average characteristic times and (b) the shape parameter of the dynamic structure factor as measured by IN11 (orange rhombs) and obtained from the simulations [blue full circles, connected with a solid line in (a)]. In (a), the time scales obtained for the different partial structure factors (empty circles) as well as for the self-correlation functions considered (squares) are also plotted. The static structure factor is shown in (a) for comparison (black dashed line).

of correlation effects (eqs 2 and 3). Thereby, all the atoms within the group have been equally considered for $\mathrm{MC}$ and $\mathrm{SG}$ groups. To avoid the additional rotational motion of the hydrogens within the dynamic window of interest, for the $\alpha$-methyl group we have taken into account only the carbon. We can see that the self-correlation times are always shorter than the corresponding collective times. The biggest difference occurs for the main-chain correlation time (factor of 3.5). This result may be related to a de Gennes narrowing, ${ }^{51}$ where the ratio between the collective and the self-time is just the static structure factor. We note, however, that in such an approach the predicted difference would be even stronger-the peak height of the mainchain correlation peak at $Q=0.8 \AA^{-1}$ is a factor of 6 higher than the asymptotic value at high $Q$. Finally, the side groups' correlation times (MG/MG, SG/SG) are much closer to their respective self-terms, thus displaying much less collectivity.

At the first structure factor peak it is quite difficult to relate the dynamics of the different partial structure functions to the observed overall relaxation, since basically all correlation functions contribute, some of them with a positive and others with negative sign (see Figure 10). For instance, the MC/MC and the MC/SG correlation functions contribute nearly identically but with opposite sign. Also, the corresponding time constants are rather similar. Figure 13a displays the net contribution of these two correlation functions to the relaxation of the structure factor at the first maximum. We realize that the nearly identical decays cancel up to a very small constant term over the full time regime of the simulation. Similarly, the SG/ SG and the MG/SG correlation functions also nearly cancel. The net result is a small negative plateau at short times, while the different time constants and amplitudes at longer times diminish the negative plateau to a very small negative value. The main effect of the observed dynamics comes from the MC/ MG and the MG/MG correlations which both reflect the backbone dynamics. To this the contribution of Figure 13b is added, which leads to an apparent slowing down. Thus, there 


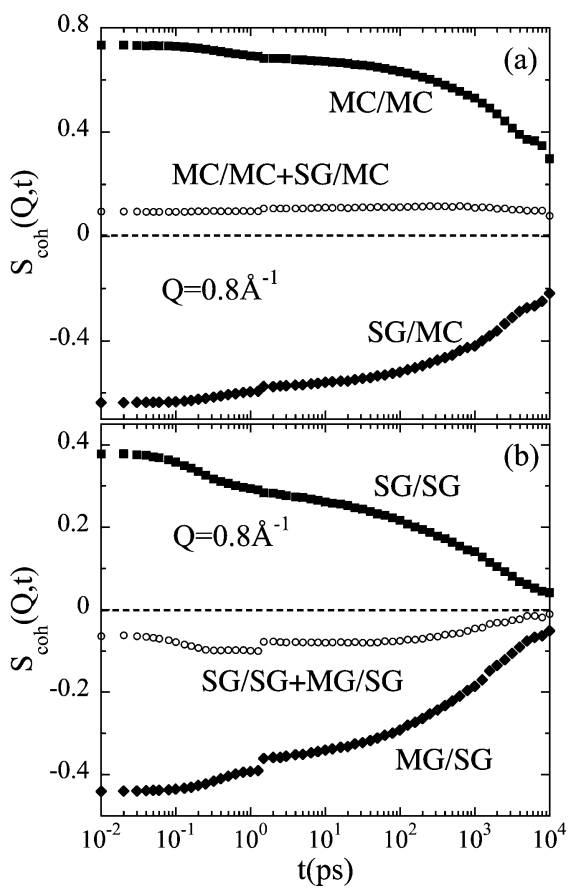

Figure 13. Partial dynamic structure factors at $Q=0.8 \AA^{-1}$ corresponding to (a) $\mathrm{MC} / \mathrm{MC}$ and $\mathrm{SG} / \mathrm{MC}$ correlations and their sum and (b) SG/SG and MG/SG correlations and their sum. The dotted lines mark the 0 value.

is no simple relation between the observed relaxation function and any particular correlation function. Indirectly, however, the relaxation of the main chain shines through, as can be seen from the closeness of the average time with the correlation times involving the main chain (see Figure 12a). Compared to the self-motions at $Q=0.8 \AA^{-1}$, the overall pair correlation function relaxes significantly more slowly, indicating some kind of de Gennes narrowing. Finally, we note that the NSE and simulation results are very close (for both the time scale and the shape).

We now turn to the higher $Q$ value, $Q=1.9 \AA^{-1}$. Here the comparison with the experiment is more simple since basically only two correlation functions contribute to the experimental average result, namely the SG/SG and the MG/MG correlation function (see Figure 10). Nevertheless, we will inspect the relaxation behavior of all correlation functions at this $Q$-point. Normalized by the corresponding static values, they are presented in Figure 11b. As can be appreciated from this figure and the values given in Table 1, the normalized amplitudes are largest for the backbone related correlations, where no fast librations are involved. The amplitudes then decrease to their lowest value for the SG/SG function, where the largest librational contributions are expected. Table 1 displays the relaxation characteristics of the different partial correlation functions including their time constants as well as their stretching exponents. As can also be seen in Figure 12a, the characteristic times are widely spread. The fastest relaxations relate to the MG/MC and SG/SG correlations, while the slowest relaxation essentially relates to $\mathrm{MC} / \mathrm{MC}$ and the $\mathrm{MC} / \mathrm{SG}$ correlation functions. Comparing the collective pair correlation functions with the respective self-terms, we realize that at this higher $Q$ the side groups' related correlation functions are identical to the self-terms. Thus, no coherency effects remain at this $Q$, and the incoherent approximation is valid. This limit is not yet reached for the $\mathrm{MC} / \mathrm{MC}$ correlation function, where a factor of 2 between the self- and the pair-correlation relaxation times is visible.
As mentioned above, at $Q=1.9 \AA^{-1}$ only the MG/MG and the SG/SG correlation functions contribute significantly to the total collective relaxation observed in an experiment. Both relaxations show very similar time constants, and their superposition is very close to the corresponding self-terms. We note that also for this $Q$ value experimental and simulation results are very close (see Figure 12a). Thus, both the observation of the incoherent scattering addressing the self-term (Figure 5) and the measured coherent scattering addressing the pair-correlation function agree with the simulation. Moreover, the good agreement between the shape of the measured and computed dynamic structure factors needs to be mentioned (see Figure 12b). Once the $65 \mathrm{~K}$ temperature shift is allowed, all these nontrivial well agreeing results reflect the overall correctness of the dynamic simulations. Furthermore, we want to note that the assumption of one single KWW function which was made when fitting the BS data is well justified in light of the simulation results: the time scale of the SG/SG correlation function is rather close to those for the self-motions of MC and MG atoms (see Table 1).

We may summarize the results on the collective processes in the following way: At the $Q$ value characteristic for the overall periodicity of the system $\left(Q=0.8 \AA^{-1}\right)$, the observed collective dynamics relates to the backbone dynamics expressed by the MG/MG and the MC/MG correlation functions and is of interchain character. ${ }^{21}$ At this point, the SG/SG correlation function decays 10 times faster than the main-chain correlations. This loss of SG/SG correlation is accompanied by a fast loss also of the SG/MG and MG/MG correlations, while at the same time the main chains stay correlated. This can only be interpreted such that the side groups lose their correlation by rotational motion around the main-chain axis, keeping the main chains locally correlated. Thus, the local structure lives much longer than the correlation of the side groups. This hypothesis is supported by the evaluation of the self-correlation function of the ester group hydrogens, where we have deduced the existence of an additional rotational motion around the main chain. It is worthy of remark that the relaxation times corresponding to the backbone collective dynamics agree quite well (see Figure 3) with the NMR correlation times recently reported ${ }^{15,16}$ for the isotropization of the orientation of the main chain in PMMA. This process has been recently evidenced in the poly $(n$-alkyl methacrylate) series and would result from the isotropic relaxation of the extended backbone conformations that retain conformational memory in these systems. It was interpreted as the genuine mechanism for the structural relaxation. ${ }^{15-17} \mathrm{We}$ note at this point that the interchain collective dynamics, which have been measured by NSE techniques at the first maximum of $S(Q)$ in different polymers, was always interpreted as the actual structural $\alpha$-relaxation. ${ }^{34}$

At $Q=1.9 \AA^{-1}$, with the exception of the MC/SG and $\mathrm{MC} /$ $\mathrm{MC}$ correlations, basically all the relaxation times are within a factor of 2. However, we can notice that both the MC/SG and MC/MC correlations have a small amplitude at this $Q$ value. Moreover, with the exception of the main chain, pair- and selfcorrelation times agree. At this length scale, all the side groups (MG, SG) appear to move independently, and the incoherent approximation should be valid. At this high $Q$ no information can be obtained on possibly correlated motions. Moreover, Figure 3 shows that the corresponding relaxation times appear to follow the dielectric $\alpha$-relaxation times reported for a similar PMMA sample. ${ }^{13}$ On the other hand, in the low-temperature range, the relaxation times obtained by NSE at the two $Q$ values considered show a weaker temperature dependence than the NMR and dielectric data. As can be seen in Figure 3, they appear 
to cross over toward the direction of the dielectric secondary $\beta$-relaxation. However, at these low temperatures the observed dynamics give raise to only a very weak decay of the structure factor involving possibly larger systematic errors (see Figure 1). In particular, the determination of the shape parameter there is subjected to a large uncertainty. Considering the $T$ dependence of the $\beta$ parameter reported for PMMA by dielectric spectroscopy, ${ }^{13}$ larger values of the average time scales are obtained for the two lowest temperatures investigated at $Q=1.9 \AA^{-1}$. The bars in Figure 3 link the values obtained in such a way with those resulting from our previous data treatment. In addition, we note that results obtained on IN11C (IN11 with a multidetector option) on deuterated PMMA below $T_{\mathrm{g}}$ (at 393 K) display a slight quasielastic decay. ${ }^{20}$ In that study the $T$ dependence of the relaxation times around $T_{\mathrm{g}}$ was found to be very similar to that of the $\beta$-process. On the other hand, we recall that $2 \mathrm{D}-\mathrm{NMR}$ data on the PMMA side-group motion by Schmidt-Rohr et al. ${ }^{11}$ showed that the main-chain and the ester side-group dynamics of this polymer are coupled. Because of the asymmetry of the carboxyl group, each $\pi$-flip of the side group is accompanied by a small rotational motion of the main chain. It could be that the weak decay of the structure factor at low temperatures which turns toward the temperature dependence of the $\beta$-relaxation relates to this coupled dynamics.

\section{Conclusions}

The combination of quasielastic neutron scattering and atomistic MD simulations well above the glass transition of PMMA has allowed to access the self- and pair-correlation functions and provided new insight into its dynamical behavior at a molecular level. The agreement between both sets of data, once a shift in temperature is allowed, is excellent, taking into account that different correlation functions at different length scales are considered simultaneously.

The study of the self-motions of hydrogens has revealed deviations from Gaussian behavior for the subdiffusive process involved in the $\alpha$-relaxation. Apparently, PMMA needs larger scales than, e.g., polyisoprene to escape the heterogeneities imposed by structural constraints. Furthermore, apart from the methyl group rotations, side-group hydrogens display additional motions with respect to the main-chain atoms. We have found strong evidence for a rotational motion of the ester side group around the main chain, which occurs on a significantly faster scale than the main-chain translational dynamics and is at the origin of the strong dynamical heterogeneity in this system.

At the first structure factor peak, the time scales for collective motion strongly depend on the molecular groups considered and are spread over more than 1 order of magnitude. The correlations involving main-chain atoms decay much more slowly than those relating side groups and seem to agree with NMR data corresponding to the isotropization of the orientation of the main chain. At these length scales the NSE experiments reflect backbone motions of interchain character, i.e., the actual structural relaxation. Furthermore, coherency effects are observed for all correlations, though the side groups display much weaker collectivity. At the second structure factor peak, coherency is lost for the side groups. Finally, when approaching the glass transition the NSE results suggest an influence of the $\beta$-process on the decay of the correlations even at the level of intermolecular correlation functions.

Acknowledgment. This research project has been supported by the European Commission NoE SoftComp, Contract NMP3CT-2004-502235. A.A., F.A., and J.C. acknowledge support from the projects MAT2004-01017 and 9/UPV00206.21513568/2001. "Donostia International Physics Center" is also acknowledged. The European Commission, NMI3 Contract RII3-CT-2003-505925, supported the experiments at the Forschungsreaktor in Jülich.

\section{References and Notes}

(1) Moreno, A. J.; Alegría, A.; Colmenero, J.; Frick, B. Phys. Rev. B 1999, 59, 5983.

(2) Moreno, A. J.; Alegría, A.; Colmenero, J.; Frick, B. Macromolecules 2001, 34, 4886.

(3) Gabrys, B.; Higgins, J. S.; Ma, K. T.; Roots, J. E. Macromolecules 1984, 17,560

(4) Floudas, G.; Higgins, J. S. Polymer 1992, 33, 4121.

(5) Arrighi, V.; Higgins, J. S.; Burgess, A. N.; Howells, W. S. Macromolecules 1995, 28, 2745.

(6) Arrighi, V.; Higgins, J. S. Physica B 1996, 226, 1.

(7) Allen, G.; Wright, C. J.; Higgins, J. S. Polymer 1974, 15, 319.

(8) Genix, A.-C.; Arbe, A.; Alvarez, F.; Colmenero, J.; Willner, L.; Richter, D. Phys. Rev. E 2005, 72, 031808 .

(9) Heijboer, J. In Physics of Non-Crystalline Solids; Prins, J. A., Ed.; North-Holland: Amsterdam, 1965; pp 231-254.

(10) McCrum, N. G.; Read, B. E.; Williams, G. Anelastic and Dielectric Effects in Polymeric Solids; Wiley: New York, 1991.

(11) Schmidt-Rohr, K.; Kulik, A. S.; Beckham, H. W.; Ohlemacher, A.: Pawelzik, U.; Boeffel, C.; Spiess, H. W. Macromolecules 1994, 27, 4733.

(12) Kuebler, S. C.; Schaefer, D. J.; Boeffel, C.; Pawelzik; Spiess, H. W. Macromolecules 1997, 30, 6597.

(13) Bergman, R.; Alvarez, F.; Alegría, A.; Colmenero, J. Non-Cryst. Solids 1998, 235-237, 580 .

(14) Beiner, M. Macromol. Rapid Commun. 2001, 22, 869.

(15) Wind, M.; Graf, R.; Heuer, A.; Spiess, H. W. Phys. Rev. Lett. 2003, 91, 155702 .

(16) Wind, M.; Graf, R.; Renker, S.; Spiess, H. W. Macromol. Chem. Phys. 2005, 206, 142 .

(17) Wind, M.; Graf, R.; Renker, S.; Spiess, H. W.; Steffen, W. J. Chem Phys. 2005, 122, 014906(1-10).

(18) Soldera, A.; Grohens, Y. Polymer 2004, 45, 1307.

(19) García Sakai, V.; Chen, C.; Maranas, J. K.; Chowdhuri, Z. Macromolecules 2004, 37, 9975.

(20) Farago, B.; Chen, C.; Maranas, J. K.; Kamath, S.; Colby, R. H.; Pasquale, A. J.; Long, T. E. Phys. Rev. E 2005, 72, 031809.

(21) Genix, A.-C.; Arbe, A.; Alvarez, F.; Colmenero, J.; Schweika, W.; Richter, D. Macromolecules 2006, 39, 3947.

(22) Springer, T. Quasielastic Neutron Scattering for the Investigation of Diffusive Motions in Solids, Liquids; Springer Tracts in Modern Physics; Springer-Verlag: Berlin, 1972; Vol. 64.

(23) Lovesey, S. W. Theory of Neutron Scattering from Condensed Matter; Clarendon Press: Oxford, 1984.

(24) Bée, M. Quasielastic Neutron Scattering; Adam Hilger: Bristol, 1988.

(25) Squires, G. L. Introduction to the Theory of Thermal Neutron Scattering; Dover Publication Inc.: New York, 1996.

(26) Mezei, F. Neutron Spin-Echo; Lecture Notes in Physics; SpringerVerlag: Heidelberg, 1980; Vol. 28.

(27) Sun, H. Macromolecules 1995, 28, 701.

(28) Sun, H.; Mumby, S. J.; Maple, J. R.; Hagler, A. T. J. Phys. Chem. 1995, 99, 5873.

(29) Sun, H. J. Phys. Chem. B 1998, 102, 7338.

(30) Theodorou, D. N.; Suter, U. W. Macromolecules 1986, 19, 139.

(31) Theodorou, D. N.; Suter, U. W. Macromolecules 1986, 19, 379.

(32) Allen, M. P.; Tildesley, D. J. Computer Simulation of Liquids; Oxford Science Publications: Oxford, 1987.

(33) Rapaport, D. C. The Art of Molecular Dynamics Simulations; Cambridge University Press: New York, 1995.

(34) Richter, D.; Monkenbusch, M.; Arbe, A.; Colmenero, J. Neutron SpinEcho Investigations on Polymer Dynamics; Adv. Polym. Sci. Vol. 174; Springer-Verlag: Berlin, 2005.

(35) Lovell, R.; Windle, A. H. Polymer 1981, 22, 175.

(36) Miller, R. L.; Boyer, R. F.; Heijboer, J. J. Polym. Sci., Polym. Phys. Ed. 1984, 22, 2021.

(37) Floudas, G.; Stepanek, P. Macromolecules 1998, 31, 6951.

(38) Arbe, A.; Colmenero, J.; Alvarez, F.; Monkenbusch, M.; Richter, D.; Farago, B.; Frick, B. Phys. Rev. E 2003,67, 051802.

(39) Narros, A.; Alvarez, F.; Arbe, A.; Colmenero, J.; Richter, D.; Farago, B. J. Chem. Phys. 2004, 121, 3282.

(40) Mossa, S.; Leonardo, R. D.; Ruocco, G.; Sampoli, M. Phys. Rev. E 2000, 62, 612 . 
(41) Smith, G. D.; Paul, W.; Monkenbusch, M.; Willner, L.; Richter, D.; Qiu, X. H.; Ediger, M. D. Macromolecules 1999, 32, 8857.

(42) Farago, B.; Arbe, A.; Colmenero, J.; Faust, R.; Buchenau, U.; Richter, D. Phys. Rev. E 2002, 65, 051803.

(43) Triolo, A.; Lechner, R. E.; Desmedt, A.; Telling, M. T. F.; Arrihi, V. Macromolecules 2002, 35, 7039.

(44) Colmenero, J.; Arbe, A.; Alvarez, F.; Narros, A.; Richter, D.; Monkenbusch, M.; Farago, B. PRAMANA J. Phys. 2004, 63, 25.

(45) Tyagi, M.; Alegría, A.; Colmenero, J. J. Chem. Phys. 2005, 122, 244909.

(46) Pérez Aparicio, R.; Arbe, A.; Colmenero, J.; Frick, B.; Willner, L.; Richter, D.; Fetters, L. J. Macromolecules 2006, 39, 1060.
(47) Arbe, A.; Colmenero, J.; Alvarez, F.; Monkenbusch, M.; Richter, D.; Farago, B.; Frick, B. Phys. Rev. Lett. 2002, 89, 245701.

(48) Colmenero, J.; Alegría, A.; Arbe, A.; Frick, B. Phys. Rev. Lett. 1992, 69, 478.

(49) Arbe, A.; Colmenero, J.; Monkenbusch, M.; Richter, D. Phys. Rev. Lett. 1998, 81, 590.

(50) Mezei, F. In Liquids, Freezing and Glass Transition; Elsevier Science Publishers: Amsterdam, 1991; pp 629-687.

(51) de Gennes, P. G. Physica 1959, 25, 825.

MA0607719 